\title{
EL PROBLEMA SANITARIO DE ESPAÑA: SANEAMIENTO Y MEDIO RURAL EN LOS PRIMEROS DECENIOS DEL SIGLO XX
}

\section{$M^{a}$ Eugenia Galiana \& Josep Bernabeu-Mestre}

\author{
Universidad de Alicante
}

\section{RESUMEN}

En los primeros decenios del siglo XX, la situación socio-sanitaria de la sociedad española fue descrita como catastrófica, tanto en el ámbito urbano como en el rural. En aquel contexto, el saneamiento del medio aparece como una de las claves del proceso de regeneración que asumió la higiene en su papel de mediadora. A través de las obras de los higienistas G. De Membrillera y Luis Muñoz Antuñano, publicadas en 1921, nos hemos acercado al análisis de la situación sanitaria de la España rural durante este periodo.

PALABRAS CLAVE: Saneamiento, salud rural, España, primeras décadas del siglo XX

\begin{abstract}
During the early decades of the 20th century, the Spanish socio-sanitary situation was described as catastrophic, both in the urban as well as in the rural setting. In that context, sanitation emerged as one of the key elements of the regeneration process taken on by hygiene in its role as mediator. Through the works of hygienists G. de Membrillera and Luis Muñoz Antuñano, published in 1921, we have approached the analysis of the sanitary situation of rural Spain during this period.
\end{abstract}

KEY WORDS: Sanitation, rural health, Spain, early decades of the 20th century.

\section{INTRODUCCIÓN}

En las primeras décadas del siglo XX la sociedad española fue equiparada, en la literatura médica de la época, a un organismo enfermo y degenerado, que precisaba tratamiento adecuado y regeneración. La situación durante este periodo se describía como catastrófica. La tuberculosis, la miseria, la prostitución, la violencia, la sífilis... y un largo etcétera de problemas de salud eran 
atribuidos a la industrialización y aparecían estrechamente unidos a las lamentables condiciones de vida y trabajo que soportaba la población ${ }^{1}$.

Sin embargo, y a pesar de que los principales problemas sociales y sanitarios se vincularon al proceso de industrialización y a la nueva sociedad urbana, no se puede olvidar que en los inicios del siglo XX la mayor parte de la población española era rural ${ }^{2}$, y no mostraba precisamente una situación sanitaria mejor que la que presentaba el mundo urbano. De hecho, si se comparan los indicadores demográfico-sanitarios de ambos grupos poblacionales, frente a la desventaja inicial que ofrecían las ciudades, se pone de manifiesto que con el paso de las décadas, los núcleos urbanos alcanzaron una posición ventajosa $^{3}$. Algunos autores explican esta situación, porque fue en las ciudades donde se produjeron los primeros intentos de mejora de la sanidad pública, y desde ahí se difundieron a las zonas rurales ${ }^{4}$. Otros, en cambio, señalan como elemento clave la desaparición de los efectos negativos de la urbanización e industrialización de las urbes 5 .

1 CAmpos, R. (1998), «Higiene y enfermedad social en la España de cambio de siglo», El Médico, 73-79, p. 73. El autor analiza cómo la industrialización, al menos en una primera etapa, contribuyó al aumento de la pobreza y la mortalidad, y cómo los higienistas ofrecieron su ciencia para solucionar los problemas sociales y sanitarios. También resulta de enorme interés el texto de CAMPOS, R; MARTINEZ, J; HuERTAS, R. (2000), Los ilegales de la naturaleza. Medicina y degeneracionismo en la España de la restauración (1876-1923), Madrid, CSIC, p. 153-193.

2 Barona Jll, Bernabeu-Mestre J, Perdiguero-Gil E. (2005), «Health Problems and Public Policies in Rural Spain (1854-1936)». En BARONA, JL, ChERrY, S. (eds.), Health and Medicine in Rural Europe (1850-1945), València, Seminari D'Estudis sobre la Ciència, 63-82, p. 63. Según Barona et al., en 1930 todavía el porcentaje de población rural en España se elevaba al $81 \%$. No obstante, los autores señalan las dificultades que muestran las estadísticas históricas españolas para delimitar entre urbano y rural. De hecho, las estadísticas oficiales consideraban urbana a la población que residía en las capitales y rural el resto de municipios. Sobre el mundo rural también hay que mencionar el texto MARTINEZ, JM (2002). El nivel de vida en la España rural, siglos XVIII-XX, Alicante, Universidad de Alicante.

3 Barona, Jll, Bernabeu-Mestre, J, Perdiguero-Gil, E (2005), p. 65.

4 Buj, A. (2003), «La vivienda salubre. El saneamiento de poblaciones (1908) en la obra del ingeniero militar Eduardo Gallego Ramos», Scripta Nova. Revista Electrónica de Geografia y ciencias sociales, VII, 146 (012). http://www.ub.es/geocrit/sn/sn-146(012).htm. También en Reher, D. y Roland, R (1998), «La Península Ibérica. España». En BARdet, J.P., DuPÂQUIER, J. (eds). Historia de las poblaciones de Europa II. La revolución demográfica, 17501914, Madrid, Síntesis, 471-494, p.489.

5 GómeZ, R. (1992). La mortalidad infantil española en el siglo XX. Madrid, CIS/Siglo XXI, p. 72. 
En un contexto marcado por la teoría regeneracionista, la Higiene española asumió un importante papel como disciplina capaz de paliar los males del país. Los higienistas se erigieron en mediadores del conflicto social, asumiendo que las desigualdades sociales estaban en el origen de la enfermedad. De este modo, se adscribieron al movimiento regeneracionista, con un discurso higiénico-moral, que centraba la regeneración en la moralización de las clases populares y en el saneamiento del medio. ${ }^{6}$ La clave de la cuestión radicaba en que el progreso de la salud pública sólo sería posible imbricando mejoras sociales con transformaciones ambientales. Los procesos morbosos se enmarcaban en un cuadro ecológico complejo en el que confluían elementos muy diversos. En una línea discursiva similar, Woods y Woodward ${ }^{7}$, aunque en un contexto geográfico y cronológico diferente (la Gran Bretaña del siglo XIX) analizan estos elementos y plantean su relación con la mortalidad. Ambos autores proponen dos grandes categorías de factores implicados en la morbilidad y mortalidad de este periodo: los factores ambientales y los factores socio-económicos, íntimamente ligados al desarrollo del capitalismo y a la industrialización.

El discurso que acabamos de esbozar no fue patrimonio exclusivo de los médicos higienistas. Los preceptos higiénico-sanitarios fueron adoptados como propios por la ingeniería sanitaria. Esta fue una disciplina, que entre los siglos XIX y XX pretendió mejorar las condiciones higiénicas de las viviendas e infraestructuras, tanto en el ámbito rural como en el urbano ${ }^{8}$, e intentó llevar a cabo políticas de carácter reformista en unas décadas marcadas por lo

6 CAMPOS, R (1998), p. 73. Por lo que se refiere al desarrollo del discurso regeneracionista y su aplicación al ámbito rural, junto a la pluralidad de lecturas que se realizó del mismo, se pueden consultar los trabajos de Mercedes Granjel: GRANJEL, M. (1999), «Regeneracionismo y Medicina en las Hurdes. Las Hurdes como problema sanitario», Medicina e Historia, 2 (4 época) y Granjel, M. (2003), Las Hurdes, el país de la leyenda, Lleida, Ed. Milenio.

7 WoOdS, R. (1984), «Mortality, poverty and the environment». En Woods, R. and WoOdWARD, J. Urban disease and mortality in nineteenth-century England. London, St. Martin's Press, Inc., 19-36, p. 20. No hay que olvidar que estos autores se refieren a la Inglaterra del S. XIX, por lo que sus conclusiones deben aplicarse con cierta precaución para el contexto español. En nuestro ámbito se puede consultar el trabajo de HUERTAS, R. (2002), «Vivir y morir en Madrid: la vivienda como factor determinante del estado de salud de la población madrileña», Asclepio, 2 (54), 253-276 y RodRíGUEZ OCAÑA, E. (2005), «Comodidad, ornamentación, higiene. Modernización urbana e higienismo en la España del siglo XIX». En Salud Pública en España. Ciencia, profesión y política, siglos XVIII-XX. Universidad de Granada, p. 157-184.

8 BuJ (2003), p. 2-3. 
que se vino en llamar el problema sanitario de España ${ }^{9}$. Se debe resaltar que los ingenieros sanitarios españoles habían adquirido una importante capacitación científica que les permitía desempeñar un papel relevante en la ordenación territorial y en la introducción de nuevas tecnologías ${ }^{10}$. Esa capacitación unida a la consideración de las cuestiones sociales y ambientales en la génesis de la situación sanitaria del país, hace que resulte de enorme interés analizar algunas de las aportaciones de este colectivo.

Para acercarnos a la visión que se ofrecía desde los presupuestos de la ingeniería sanitaria, hemos analizado las obras del ingeniero Francisco G. de Membrillera $^{11}$ y del médico Luis Muñoz de Antuñano ${ }^{12}$, premiadas por el Instituto de Ingenieros Civiles en el concurso "García Faria», convocatoria dedicada a galardonar los mejores estudios sobre el problema sanitario de España y los medios para resolverlo. El interés que nos ha despertado la lectura de ambos textos, sobre todo en el análisis que llevan a cabo del entorno rural, y el hecho de enmarcarse en el contexto que hemos expuesto en los

9 A través de un análisis demográfico y de causas de muerte, el problema sanitario español, fue descrito por los higienistas de finales del XIX y principios del XX, como una situación caracterizada por altas cifras de natalidad y mortalidad (sobre todo mortalidad infantil), lo que se traducía en un aumento poblacional pequeño. Las principales causas de muerte eran las denominadas enfermedades evitables, que causaban estragos entre la población por la falta de una buena administración sanitaria y por falta de cultura higiénica de la población. G. DE MEMBRILLERA, F. (1921), El problema sanitario de España. Madrid, Sarriá, J. Viñals editor, p. 19-21. ReVENGA, R. (1904) La muerte en España, Madrid, Imprenta de la prensa de Madrid.

10 Buj (2003), p. 2.

11 Ingeniero y escritor. Efectuó numerosos viajes por distintas provincias de España, lo que le permitió observar personalmente las circunstancias sanitarias de los pueblos españoles. En algunas regiones que no pudo visitar, contactó con «personas competentes» de la zona, que le facilitaron la información que utilizaría para elaborar su trabajo: G. DE MEMBRILLERA, F. (1921), El problema sanitario de España. Exceso de mortalidad. Causas que la producen. Influencia de la Higiene en la economía nacional. Medidas de gobierno y acción de los ciudadanos para higienizar España y arrebatar a la muerte más de 100.000 vidas por año, Madrid, Sarriá, J. Viñals editor.

12 Médico militar e higienista, posicionado políticamente en las doctrinas socialistas y sindicalistas y cuya obra, MUÑOZ, L (1921), Saneamiento e higienización de España, Madrid, Sucesores de Rivadeneyra, recoge de forma pormenorizada la problemática situación sanitaria de la España del momento. Se debe resaltar el exhaustivo trabajo llevado a cabo por estos autores. Ambos textos nos muestran una gran variedad de datos que hacen pensar en la gran labor de indagación y recopilación que desarrollaron. Las reflexiones y el análisis que acompaña a esta información, también son dignos de mención y nos aproximan a lo que podríamos denominar, utilizando una terminología mas actual, un acertado diagnostico de salud de la población española, particularmente la rural, en las primeras décadas del siglo XX. 
párrafos anteriores, nos ha hecho elegirlos para acercarnos al análisis de la percepción ambientalista en el abordaje de los problemas de salud en la España de principios del siglo XX.

Nos centráremos, a continuación, en describir el estado de salud de la población rural española y los principales problemas sanitarios que preocupaban a los higienistas y, en particular, a los ingenieros sanitarios: causas de la insalubridad, situación demográfica, vivienda, ciclo del agua, paludismo y anquilostomiasis, como enfermedades rurales vinculadas al entorno, y la infancia como población especialmente vulnerable. Concluiremos este análisis revisando qué acciones fueron propuestas para modificar esta situación y mejorar las condiciones de vida de la población. Todo esto nos permitirá aproximarnos a la situación sanitaria de la España rural de las primeras décadas del siglo XX a través de la idea de saneamiento ambiental y valorar la significación histórica de los textos analizados.

\section{EL ESTADO DE SALUD DE LA POBLACIÓN RURAL ESPAÑOLA}

La situación sanitaria de la población rural española de principios de siglo era ciertamente precaria. Los escasos datos demográficos y el análisis de los elementos implicados en la salud y en la vida de la población, nos muestran una sociedad con enormes deficiencias sanitarias, económicas y sociales ${ }^{13}$. Las altas cifras de mortalidad y morbilidad fueron vinculadas a la situación de insalubridad que afectaba a todos los ámbitos de la vida de las personas. Los trabajos analizados así lo reflejan, y señalan tres elementos que contribuyeron a perpetuar esta situación ${ }^{14}$.

Por un lado, se apuntaba como elemento fundamental, la irresponsabilidad de los poderes públicos, que no ponían el esfuerzo ni los recursos necesarios para atajar el problema. De otro, la incultura y falta de moralidad de la población, a la que se culpabilizaba de sus malas condiciones de vida. De forma trasversal, pues estaba presente de un modo u otro en los dos anteriores, apa-

13 Pascua, M. (1934), La mortalidad infantil en España, Madrid, Departamento de Estadísticas Sanitarias de la Dirección General de Sanidad, p. 51-55. El autor reflexiona sobre la dificultad de obtener datos demográficos de calidad sobre la población rural y compara la mortalidad infantil rural y urbana durante el periodo 1910-1932. REVENGA (1904), p. 27-58, también analiza, entre otras cuestiones, la mortalidad de principios de siglo por capitales y provincias. Ver además, GÓMEZ (1992), p. 72.

14 G. DE Membrillera (1921), p. 20-21; MUÑOZ (1921), p.114. 
recía un tercer elemento, vinculado a las deficientes condiciones de vida de la población rural y que podríamos denominar la explicación socioeconómica de los problemas de insalubridad.

La falta de actuación de los poderes públicos, se traducía en la deficiente inversión económica en Sanidad e Higiene ${ }^{15}$. Los autores eran conscientes de que una buena administración sanitaria con un presupuesto sanitario adecuado, era una inversión de futuro, pues la enfermedad y la muerte ocasionaban graves perjuicios a la economía nacional. Faltaba presupuesto, pero también faltaba conciencia pública y formación de los gobernantes. La incultura sanitaria no era patrimonio exclusivo de los obreros y campesinos, sino que reinaba en todas las clases sociales, incluida la clase dirigente ${ }^{16}$.

El segundo de los elementos, la incultura y la falta de moralidad de la población, se convertiría en uno de los objetivos de la acción sanitaria. La falta de higiene pública y privada observada por los ciudadanos, el incumplimiento de la ley y la idiosincrasia higiénica de los habitantes se apuntaron como las principales causas de la miseria y el pauperismo ${ }^{17}$. La degeneración física y moral de las clases populares y sus nocivos hábitos de vida se situaron en el origen de la enfermedad, lo que contribuyó a culpabilizar de su propia situación a las personas y clases sociales que la padecían ${ }^{18}$, llegando a criminalizar sus conductas ${ }^{19}$.

El tercero de los elementos podría resumirse en la frase de Ángel Fernández Caro, prologuista de la obra de Membrillera, «[...] no se impide (la enfermedad), sino mejorando las condiciones de la existencia, regulando la vida de trabajo, abaratando las subsistencias, [...], el derecho a la salud y a la vida

15 En España, en el año 1921, la Sanidad Pública tenía un presupuesto anual de 2 millones de pesetas que, salvando la distancia temporal, era a todas luces insuficiente. Comparaba Muñoz Antuñano, lamentándose de ello, este escaso presupuesto con la dotación económica para sanidad de Framinghan, ciudad estadounidense de 15.000 habitantes, y que disponía de 15.000 dólares anuales para cubrir sus necesidades de salud [MUÑOZ (1921), p. 9-10]

16 Fernandez, A., prólogo de la obra de G. DE MEMBriLlera (1921), p. I

17 G. DE MEMBRILlera (1921), p. 81.

18 CAMPOS (1998), p. 75.

19 El concepto de enfermedad como plaga social, que en esta época estuvo teñido de términos como alcoholismo, prostitución o miseria, sobre todo en el mundo urbano, aparece algo más desdibujado en el mundo rural. En los textos analizados, toma más cuerpo la idea de la incultura que la de la inmoralidad, y la culpabilización de los individuos, aunque aparece, no tiene la misma intensidad que cuando se hace referencia al mundo urbano. Sobre el problema de la culpabilización se puede consultar: Perdiguero-Gil, E, Bernabeu-Mestre, J. (1997), «Burlarse de lo cómico nacido de la tontería humana: el papel otorgado a la población por la divulgación sanitaria durante la Restauración». En MonTIEL, L, PORRAS, I. (eds.) De la Responsabilidad Individual a la Culpabilización de la Víctima. Madrid, Doce Calles, p. 55-66. 
es patrimonio de la Humanidad entera» ${ }^{20}$. El estatus socioeconómico marcaba importantes diferencias entre las personas. No tenía la misma esperanza de vida el rico que el pobre; cincuenta y treinta años respectivamente. Tampoco los datos de mortalidad eran los mismos; había mayor número de víctimas entre la clase proletaria que en la acomodada. La miseria, indigencia y falta de recursos para cubrir necesidades básicas como la alimentación o las medicinas, causaban estragos entre las clases populares. La reivindicación de un reparto igualitario de los bienes, sobre todo en lo relativo a la propiedad de la tierra, cobraba fuerza en el mundo rural. Se hablaba de la necesidad de incorporar a las leyes una nueva perspectiva en las relaciones de dominio de los patronos sobre los obreros: había que «desvincular, desenfeudar, descastar el Poder» ${ }^{21}$. Por todo ello, era necesario modificar la organización de la propiedad rustica y la forma de arrendamiento. Mejorar las condiciones de vida del campesino, era mejorar su salud y la de los suyos.

\subsection{Aproximación demográfica}

Se quejaba Luis Muñoz de Antuñano, y no era el único, de la ausencia de fuentes de información, de datos y estadísticas que le permitieran llevar a cabo una labor seria y documentada en la descripción de la situación sanitaria del país $^{22}$. Pero además, si lo que se pretendía era tener información de la situación de la sanidad rural, el problema se agravaba ${ }^{23}$. Otros autores también han subrayado este problema. Según Rodríguez Ocaña, hasta 1899 no aparecieron los primeros intentos gubernamentales de consolidar un sistema de registro sanitario fiable, ausente hasta ese momento. La iniciativa fracasó en un primer momento al intentar que fuesen los médicos titulares los promotores del registro.

20 FERnÁndeZ, prólogo de la obra de G. DE MEMBrillera (1921), p. III.

21 MuÑOZ (1921), p. 136.

22 Según MuÑoz (1921), p. 1, personalidades como D. José Canalejas, D. Juan de la Cierva y otros políticos, hombres de estado, de ciencia, se habían lamentado de ello en numerosas ocasiones. Significativas son las palabras de Canalejas: «Cuando, como Ministro, como gobernante, tengo que decretar o proponer, me desespero, careciendo incluso de fuentes de información que sirvan de norma a tan altas gestiones».

23 MuÑOZ (1921), p.118, añadía «Por tal razón debe acometerse la completa organización de los servicios, reuniendo los datos aislados [...], aportando otros nuevos y modificando y completando los existentes. Así podremos saber [...] el índice palúdico español, las comarcas atacadas, las aguas de que disponemos, sus medios de conducción y distribución, [...] la alimentación y el trabajo del habitante del campo, las epidemias y las endemias[...], y tantos otros datos, que juntos constituirían el inventario de nuestra sanidad rural.» 
Posteriormente se asignó la tarea a los Inspectores provinciales de Sanidad, con igual suerte, lo que supuso la renuncia a esa vía sanitaria estadística. Por todo ello, no quedó más remedio que contentarse con los datos demográficos disponibles a partir de 1902 y que eran suministrados por el Instituto Geográfico y Catastral anualmente, y que son los que utiliza Membrillera para su descripción demográfica, concretamente los correspondientes al periodo que va de 1911 a 1917. Probablemente debido a este déficit y a la riqueza de datos que este último autor aporta, podría pensarse que recurrió a otras fuentes para recopilar parte de la información que figura en su trabajo. De hecho presenta datos acerca de la situación de salubridad de las capitales de provincia que podrían haberse obtenido a través de un cuestionario elaborado por el mismo ${ }^{24}$.

A pesar de las dificultades, en las obras analizadas, los autores elaboraron una amplia descripción demográfica que nos da idea de la situación en que se encontraba el país. España contaba en aquel momento con 19.524.808 habitantes. La tasa de natalidad media era de 30.5 por 1000 habitantes, cifra que fue gradualmente disminuyendo a lo largo del periodo estudiado y en la que predominaba el nacimiento de varones sobre el de mujeres. Al analizar su distribución geográfica, se observa un mayor número de nacimientos en las pequeñas ciudades y pueblos rurales que en las grandes urbes. Con respecto a la mortalidad, se barajaba la cifra de 22.04 por cada 1000 habitantes, con un comportamiento diferencial según los grupos de edad, contribuyendo en gran medida las dos primeras edades, es decir el grupo de 0 a 5 años. El predominio por sexos seguía siendo para los varones; al igual que nacían más hombres también morían más hombres. Sin embargo con respecto a la distribución geográfica no se producía el mismo fenómeno, y así en provincias moría menor número de personas que en las capitales. Los autores no aportan cifras de mortalidad infantil diferenciadas en población urbana y rural, lo que nos permitiría hacer una mejor comparación, pero los datos disponibles nos remiten a las primeras fases del proceso de transformación demográfico y sanitario de la sociedad española en el primer tercio del siglo XX. Como mencionamos en párrafos anteriores, estas primeras etapas se caracterizaron por una peor situación para el entorno urbano, que en comparación con el entorno

24 RodríGUEZ OCAÑA, E. (1996), «La encuesta sanitaria como contribución original de Philipp Hauser a la salud pública española». En CARRILlo, JL. (ed), Entre Sevilla y Madrid. Estudio sobre Hauser y su entorno. Sevilla, Universidad de Sevilla, 195-210, p. 203 Así lo plantea el autor cuando al estudiar la actividad científica de $\mathrm{Ph}$. Hauser, médico higienista de finales del XIX y principios del XX, menciona la obra de G. de Membrillera como ejemplo de una interesante iniciativa que llevó a cabo indagaciones a través de cuestionarios de elaboración propia, aunque G. de Membrillera no lo mencione de forma explicita. 
rural presentaba tasas de natalidad más bajas y tasas de mortalidad por todas las causas más altas. En etapas posteriores esto fue cambiando y los núcleos urbanos, sobre todo los que vivieron de forma más intensa el proceso de industrialización, consiguieron una mejor situación ${ }^{25}$.

Por lo que respecta a las causas de muerte, aunque tampoco disponemos de datos diferenciales según contexto urbano o rural, en las obras analizadas se pone el énfasis en las enfermedades infecciosas y evitables. En las tablas siguientes se reproducen los datos de mortalidad por causas de muerte y su evolución, datos coherentes con la dinámica demográfica española de finales del XIX y principios del $\mathrm{XX}^{26}$.

\section{TABLA 1. CONTRIBUCIÓN DE LAS DIFERENTES ENFERMEDADES A LA MORTALIDAD. 1906-1910 27}

\begin{tabular}{|l|c|l|c|}
\hline ENFERMEDADES & $\begin{array}{l}\text { Promedio anual por } \\
\text { 1000 defunciones }\end{array}$ & ENFERMEDADES & $\begin{array}{l}\text { Promedio anual por } \\
1000 \text { defunciones }\end{array}$ \\
\hline $\begin{array}{l}\text { Diarrea y enteritis } \\
\text { (menores de dos años) }\end{array}$ & 95 & Bronquitis crónica & 26 \\
\hline $\begin{array}{l}\text { Hemorragia y reblan- } \\
\text { decimiento cerebrales }\end{array}$ & 65 & Gripe & 25 \\
\hline $\begin{array}{l}\text { Enfermedades orgáni- } \\
\text { cas del corazón }\end{array}$ & 65 & $\begin{array}{l}\text { Cáncer y otros tumores } \\
\text { malignos }\end{array}$ & 20 \\
\hline $\begin{array}{l}\text { Tuberculosis de los } \\
\text { pulmones }\end{array}$ & 55 & $\begin{array}{l}\text { Nefritis aguda, mal de } \\
\text { Bright }\end{array}$ & 17 \\
\hline Bronquitis aguda & 54 & Sarampión & 16 \\
\hline $\begin{array}{l}\text { Otras enfermedades } \\
\text { del apto. respiratorio } \\
\text { (excepto tisis) }\end{array}$ & 53 & $\begin{array}{l}\text { Muertes violentas (ex- } \\
\text { cepto suicidio) }\end{array}$ & 13 \\
\hline Meningitis simple & 43 & $\begin{array}{l}\text { Fiebre tif. (tifus abdo- } \\
\text { minal) }\end{array}$ & 12 \\
\hline Senilidad & 38 & $\begin{array}{l}\text { Afras tuberculosis } \\
\text { go (excepto cáncer) }\end{array}$ & 11 \\
\hline Neumonía & 37 & \multicolumn{2}{|l}{} \\
\hline $\begin{array}{l}\text { Debilidad congénita y } \\
\text { vicios de conformación }\end{array}$ & \multicolumn{2}{|l}{} \\
\hline
\end{tabular}

25 Barona; Bernabeu-Mestre; Perdiguero-Gil (2005), p. 128.

26 Robles, E, García, F, Bernabeu-Mestre, J., (1996), «La transición sanitaria en España desde 1900 a 1990», Revista Española de Salud Pública, 221-233, p. 221-223.

27 G. DE MEMBrillera (1921), p. 5 
TABLA 2. EVOLUCIÓN DE LA MORTALIDAD POR ENFERMEDADES INFECCIOSAS. 1906-1910 28

\begin{tabular}{|l|r|r|r|r|r|c|}
\hline $\begin{array}{l}\text { ENFERMEDADES INFEC- } \\
\text { CIOSAS }\end{array}$ & 1906 & 1907 & 1908 & 1909 & 1910 & $\begin{array}{c}\text { PROMEDIO } \\
\text { ANUAL }\end{array}$ \\
\hline Fiebre tifoidea & 16.17 & 14.46 & 14.77 & 11.54 & 10.05 & 13 \\
\hline Viruela & 8.70 & 6.86 & 6.07 & 8.43 & 4.61 & 7 \\
\hline Sarampión & 17.43 & 14.48 & 15.57 & 16.60 & 15.07 & 16 \\
\hline Escarlatina & 2.80 & 4.99 & 4.75 & 3.69 & 3.55 & 4 \\
\hline Coqueluche & 8.88 & 7.20 & 10.50 & 7.60 & 5.46 & 8 \\
\hline Difteria y crup & 6.79 & 7.70 & 8.52 & 9.11 & 9.29 & 8 \\
\hline Gripe & 20.99 & 39.32 & 19.65 & 22.37 & 21.39 & 25 \\
\hline Tuberculosis pulmonar & 55.11 & 57.23 & 57.80 & 53.02 & 53.88 & 55 \\
\hline Total & 136.8 & 152.2 & 137.6 & 132.3 & 123.2 & 136 \\
\hline
\end{tabular}

Al comparar estos datos con los de otros países ${ }^{29}$, G. De Membrillera subraya que España superaba tanto en natalidad como en mortalidad a la mayoría de las naciones, siendo esto la causa de su escaso incremento poblacional anual. Este exceso de mortalidad se daba principalmente entre los menores de 5 años, situación que se repetía en la mayoría de las naciones analizadas, pero que colocaba a nuestro país entre los más desfavorecidos en mortalidad infantil ${ }^{30}$. Las principales causas de muerte que superaban en proporción a los casos de otras naciones eran la fiebre tifoidea, las fiebres intermitentes, viruela, sarampión, gripe, meningitis, bronquitis, tuberculosis, tos ferina y difteria, es decir, las enfermedades consideradas «evitables», cuya prevención estaba resuelta por la Medicina, pero para las que no se había articulado una actuación preventiva eficaz ${ }^{31}$

28 G. DE MEMBRILLERA (1921), p. 6

29 G. DE Membrillera (1921), p. 11. Datos correspondientes al decenio de 1901 a 1910 , publicados por el Instituto Geográfico y Estadístico.

30 Pascua (1934), p. 3-10. ReVenga (1904) p. 59-80)

31 En el trabajo de Porras Gallo, M.I. (1994), «La lucha contra las enfermedades «evitables» en España y la pandemia de la gripe de 1918-19», Dynamis, 14, 159-185, la autora pone de manifiesto los intentos por conformar esta actuación a través de distintas propuestas legislativas de los médicos españoles: el Real Decreto de 10 de Enero de 1919 de prevención de enfermedades contagiosas, el proyecto de Ley de epidemias (1914), el proyecto de Ley sobre profilaxis pública de las enfermedades infecciosas (julio de 1919); el denominado de igual modo pero presentado en Julio de 1919; y el proyecto de Ley de profilaxis pública de 
Con estos datos, la situación no era muy prometedora; indicando ambos autores que «nos encontrábamos ante un verdadero problema nacional» $»^{32}$. Se imponía la necesidad de analizar en detalle la situación sanitaria y dejar de eludir la obligación de proponer medidas de mejora que abordaran el problema en su totalidad.

\subsection{La vivienda rural}

«Suele tener dos pisos: el superior, y la planta baja, compuesta de dos departamentos, utilizándose uno para comedor, cocina y deposito de los aperos de labranza, y el otro de cuadra [...]; si subís, encontrareis dos o tres lóbregas habitaciones, y dos únicas y míseras camas, una para dormir los padres, y en la otra todos los demás hijos [...] las ventanas suelen ser reducidas ,y en invierno permanecen casi siempre cerradas [...] el retrete o no existe, o está en comunicación directa con el dormitorio, y en voladizo para verter sobre el estercolero; en donde el estiércol, fermentando, aromatiza a los habitantes. Además, éstos aspiran, el aire enrarecido por la respiración y secreciones del ganado, que penetran en la habitación, que es templada con esta calefacción insana»» ${ }^{33}$.

Así describía G. de Membrillera la situación de la vivienda rural española. El esfuerzo por describir el «verdadero estado sanitario de España», permitió tomar conciencia de un hecho: las viviendas rurales españolas no cumplían los requisitos mínimos de habitabilidad. Esta conclusión se pudo formular después de un importante esfuerzo de recopilación de datos, porque, si escasos eran los datos demográficos, más lo eran los relativos a saneamiento, infraestructuras y condiciones sanitarias de las viviendas rurales. Estas eran las condiciones, a pesar de que en la Reglamentación de la Inspección de Sanidad del Campo, se prescribía el estudio de las viviendas agrícolas y obreras del campo ${ }^{34}$.

A pesar de estas limitaciones, las obras analizadas, no se ciñen únicamente a la valoración del interior de la morada y sus dependencias. Amplían su vi-

enfermedades evitables (1922). Sin embargo, la pandemia de gripe de 1918-19 fue, en palabras de la autora, «un serio revés a la arrogante Medicina de la época», que no pudo abordar el problema desde los principios de la Bacteriología, y que además, puso de relieve la precariedad de las instituciones sanitarias y sociales de nuestro país». También en el texto de G. DE MEMBRILLERA (1921), p. 19-20, se utiliza la misma terminología para hacer referencia a las enfermedades médicamente prevenibles.

32 G. DE MEMBriLlera (1921), p. V.

33 G. DE MEMBRILLERA (1921), p. 24-25.

34 MuÑOZ (1921), p. 121 
sión e incorporan el análisis de una serie de elementos -emplazamiento, aireación, estado de calles y caminos, etc.--, que por extensión, también debían ser tenidos en cuenta por incidir de forma directa en la salud de sus moradores ${ }^{35}$.

En general, las condiciones de las viviendas rurales eran muy deficientes. Sin embargo, parece que había algunas diferencias por regiones. No era lo mismo vivir en un pueblo del litoral, con un clima benigno, y probablemente, con mayor acceso a las escasas medidas higiénicas del momento, que en una pequeña aldea aislada del interior del país, en las que ni siquiera se usaba la cal para el blanqueo de fachadas, - lo que procuraba una imagen de mayor limpieza-, y en las que la higiene general, tanto de personas como de enseres, era mas deficiente ${ }^{36}$.

Mejorar las condiciones de la vivienda era mejorar la salud de sus habitantes. En las condiciones descritas, las casas de los campesinos, no sólo no protegían a sus moradores contra los riesgos sanitarios, sino que eran fuente de enfermedad y muerte. Se vincula de forma repetida vivienda insalubre y enfermedad, algo que había estado presente a lo largo del siglo XIX ${ }^{37}$, pero que toma especial relevancia en la España del cambio de siglo. La acción higiénica, recaía en el saneamiento del medio, entendido este último desde un punto de vista físico y social, y la vivienda puede considerarse como el principal exponente de esta concepción, al depender su configuración y condiciones tanto de cuestiones físicas como sociales. Era preciso cambiar el modelo y para ello los autores esbozan una propuesta de vivienda rural higiénica:

35 Esta idea no es exclusiva de G. de Membrillera. En el trabajo de BERnABEU-MeStre J. (2006), «Medio urbano y salud en el proceso de modernización: los trabajos de la Academia de Higiene de Cataluña, 1892-1922». En BeAscoecheA, J.M., GonZÁlez, M. y Novo, P.A. (eds), La ciudad contemporánea, espacio y sociedad, Bilbao, Universidad del País Vasco, 183-200, p. 193, al analizar los resultados de una encuesta sobre el estado sanitario de Cataluña (1903-1906), realizada por la Academia, se identificaban entre las causas de insalubridad de las viviendas, las causas exteriores, que fueron clasificadas como «envolventes» o «de sustentación». De hecho, esta concepción, no se aleja en exceso del concepto actual de vivienda, que amplía sus límites hasta el entorno próximo del individuo. La palabra vivienda se extiende a todo cuanto haya en las cercanías, es decir paseos, caminos, calles, espacios abiertos, centros sanitarios, escuelas o servicios sociales de la vecindad. (BRITISH MEDICAL ASSOCIATION (2003). Housing and Health: building for the future, London, BMA, p. 41.)

36 G. DE MEMBRILLERA (1921), p. 24.

37 FresqueT, JL. (1992), «La higiene municipal en la obra de Francisco Méndez Álvaro (1806-1883)». En CAPEL, H; LÓPEZ, J.M., y PARDO, J. (coords.). Ciencia e Ideología en la ciudad. I Coloquio Interdepartamental. Valencia, Generalitat Valenciana, Conselleria d'Obres Públiques, Urbanismo i Transport, 265-278. 
«Todas las edificaciones rurales deben ser sometidas a un reglamento social y a un bien entendido saneamiento; aguas, retretes, lavaderos, inmundicias, establos,... [...] La casa agrícola debe separar los animales de las habitaciones humanas, [...]; el pozo debe ser cubierto, si le hay, y los retretes se construirán obligatoriamente» ${ }^{38}$.

\subsection{Los problemas relacionados con el ciclo del agua}

Las deficiencias relacionadas con el uso del agua fueron uno de los elementos que más claramente se relacionaron con los problemas de salud. Los trabajos que estamos analizando hacen especial hincapié en esta cuestión, como veremos a continuación. Antes de ello, vale la pena mencionar dos interesantes iniciativas que, en este mismo periodo, analizaron la relación entre insalubridad, enfermedad y condiciones del agua. Por un lado, el estudio llevado a cabo por la Academia de Higiene de Cataluña, que utilizó datos recogidos a través de una encuesta, ya citada en párrafos anteriores, y que situaba las deficiencias relacionadas con el ciclo del agua entre los principales determinantes de salud de los catalanes ${ }^{39}$. La segunda de las iniciativas, algo anterior en el tiempo, la llevó a cabo la Sociedad Internacional de Aguas Potables, fundada a raíz del Congreso Internacional de Higiene de Paris (1878) y que tuvo una adscripción colectiva de los médicos andaluces reunidos en el II Congreso Regional de Ciencias Médicas celebrado en Cádiz en 1879. Desde esta sociedad, y para iniciar su labor en nuestro país, se remitió una encuesta a todos los médicos españoles sobre la situación del agua potable en su jurisdicción y su relación con las epidemias o enfermedades de la localidad ${ }^{40}$.

El tema del agua, por tanto, se situaba entre las principales preocupaciones de los higienistas. Era de sobra conocida su relación directa con las enferme-

38 MUÑOZ (1921), p.128

39 BERnABEu-Mestre (2005), p. 293-295. De hecho, en esta encuesta, que tenia el objetivo de aproximarse al estado sanitario de Cataluña, y que solicitaba información a todos los médicos titulares, se hacia especial énfasis en esta cuestión. Tanto es así, que la recogida de información, que se clasificaba en ocho apartados, reservaba de forma exclusiva uno de ellos a las cuestiones relacionadas con el agua. Además, cuando se analizaron los resultados, los autores atribuyeron a la ausencia de alcantarillado (en el $70 \%$ de los municipios) y a la escasez e impureza del agua potable (en el 49\%), las causas de morbilidad y mortalidad,

40 En el trabajo de RoDRíGUEZ OCAÑA (1996), p. 201-203, se especifica que la encuesta constaba de cinco preguntas sobre las fuentes de agua, su depósito y posible contaminación, así como con su relación con las enfermedades de la zona. Según Rodríguez Ocaña, «los resultados de esta encuesta sobre aguas no han sido referidos ni comentados en ningún texto conocido, por lo que lo más probable es que careciera de contestaciones suficientes». 
dades infecciosas ${ }^{41}$, y a pesar de ello pocos esfuerzos se hacían para mejorar la situación en España. La Ley de Aguas vigente en este periodo, reguladora de los abastecimientos en calidad y cantidad, era la del 13 de Junio de 1879, anticuada en comparación con otros países europeos, e insuficiente para mejorar la situación ${ }^{42}$. Las deficiencias de la Ley se podían resumir, según Antuñano, en dos cuestiones. Por un lado, no reflejaba el espíritu moderno de la legislación vigente en los demás países, al no incluir los conocimientos mas actualizados del origen hídrico de las enfermedades, así como el de los progresos y necesidades industriales. Por otro, y en relación a cuestiones más prácticas, cifraba en 20 litros por habitante y día la cantidad agua potable que debían proveer los abastecimientos públicos, cifra claramente insuficiente para las necesidades del momento:

«dadas nuestras actuales costumbres y necesidades, no parece que debe ser exagerada la cifra de 50 litros por día y habitante en la mayoría de poblaciones menores de 10000 almas, y de 100 litros en adelante para las restantes» ${ }^{43}$.

Pero además del aumento de la cantidad, era indispensable proteger todo el sistema de abastecimiento con una buena conducción que asegurara unas condiciones de calidad mínimas. Muchas de las aguas se filtraban y perdían en el trayecto y otras se contaminaban sin remedio. De esta forma era difícil que el agua reuniera las tres condiciones que se consideraban básicas para su consumo: potabilidad, suficiencia y pureza. Las dos primeras no eran un problema, pues se disponía de aguas procedentes de manantiales, potables y sufi-

41 No obstante, «por si acaso se dudara de ello», MUÑOZ (1921), p. 195, ilustraba su escrito con datos procedentes de la campaña alemana contra la fiebre tifoidea, y resaltaba que de 5889 casos de enfermedad, el contagio por agua se había producido en 399, ocupando el segundo lugar en motivo de contagio, tras el contagio directo.

42 La gestión del suministro de aguas y su vigilancia, así como la evacuación de aguas residuales fue, durante la segunda mitad del S. XIX y principios del S. XX, una responsabilidad municipal. Perdiguero, E. (1997), «Problemas de salud e higiene en el ámbito local». En: Bernabeu-Meste, J., Esplugues, JX., Robles, E. Higiene I Salubritat en els municipis valencians (1813-1939), Benissa, Seminari d'Estudis sobre la Ciencia. Institut d'Estudis Comarcals de la Marina Alta, 17-41, p. 25-30.

43 MUÑOZ (1921), p. 200. Para poder establecer una comparación, recordaremos que la OMS recomienda en la actualidad que la cantidad suministrada no sea inferior a 90 1/habitante/día para poblaciones rurales, $125 \mathrm{l} / \mathrm{habitante/día} \mathrm{para} \mathrm{poblaciones} \mathrm{menores} \mathrm{de}$ 500000 habitantes y 250 1/habitante/día para poblaciones de más de 500000 habitantes. ESPIGARes M, Moreno O, FernÁndez-Creuet M. (2001), «Aspectos sanitarios del agua». En PIÉDrola, G. Medicina Preventiva y Salud Pública. Barcelona, Masson, 301-329, p. 301. 
cientes. En realidad, el problema se centraba en la «pureza», es decir en su contaminación, lo que señalaba a los deficientes sistemas de conducción, escasamente protegidos y en ocasiones ausentes, como la causa de la misma. España contaba con suficientes recursos naturales, pero la dejadez y falta de protección por parte del Estado, hacia que estos recursos se «impurificaran del modo más lastimoso» ${ }^{44}$.

El 27 de Marzo de 1914, se promulgó un Real Decreto, que consignaba créditos para auxiliar a los abastecimientos de aguas potables de las pequeñas poblaciones. Aquella iniciativa no resolvía el problema con la amplitud que requería. Era necesario remediar la situación a través de reformas legislativas de mayor peso ${ }^{45}$.

2.4. La insalubridad ambiental como origen de la enfermedad: paludismo $\mathrm{y}$ anquilostomiasis en el mundo rural

\subsubsection{Paludismo}

El paludismo ha estado desde la antigüedad vinculado al mundo rural. El descubrimiento del plasmodio por Alphonse Laveran se produjo en 1880, pero no fue hasta 1902, cuando se dispuso de la información adecuada para iniciar la acción sanitaria de lucha contra la enfermedad.

En España, la campaña antipalúdica ${ }^{46}$, que se inició con cierto retraso en relación con otros países, puede considerarse como el origen de la sanidad rural. La primera acción sanitaria antipalúdica fue la realizada en Río Tinto, por Ian McDonald, que consiguió, entre 1903 y 1907, que los episodios de paludismo dejaran de presentarse. Pero, probablemente, la contribución más relevante fue la de Gustavo Pittaluga quien, desde algunos de los cargos que desempeño en

\footnotetext{
44 MuÑOZ (1921), p. 117

45 MuÑOZ (1921), p. 203

46 Sobre el estudio histórico del proceso de lucha contra el paludismo en la España del siglo XX, resulta fundamental la consulta del trabajo de RoDRíGUEZ, E.; BALLESTER, R.; Perdiguero-Gil, E., MedinA, R. y Molero, J. (2003), La acción médico-social contra el paludismo en la España metropolitana y colonial del siglo XX. CSIC, Madrid. Resulta de interés para el trabajo que nos ocupa señalar que, según los autores, el estudio del paludismo, supuso un replanteamiento del papel del medio ambiente en la génesis de la enfermedad, que adoptó un sentido más biológico. Paralelamente, también se vinculó a la teoría telúrica al defender la presencia necesaria de determinadas condiciones ambientales, el denominado «ambiente palúdico».
} 
la Sanidad oficial, orientó y estructuró las investigaciones científicas y los programas de actuación frente a la enfermedad en nuestro país ${ }^{47}$. Ambos autores, junto a Pi i Sunyer y Gabriel Carrió, son citados por Muñoz Antuñano como «los que primeramente se ocuparon en España de la cuestión» ${ }^{48}$.

En relación con los datos sobre paludismo en España, Antuñano recogía en su trabajo parte de un informe que en 1918 emitió el Ministerio de Fomento, concretamente la Inspección para el Saneamiento del Campo, y que formaba parte del Inventario del Paludismo, publicado anualmente por aquel organismo. Los datos, que incluían una valoración económica del problema, dibujaban una situación, cuando menos, preocupante:

PALUDISMO EN ESPAÑA - TOTALES GENERALES
\begin{tabular}{|l|r|}
\hline 9 & \\
\hline Número de términos municipales en España & 9.261 \\
\hline Ídem de íd. Íd. Palúdicos de ídem & 1.818 \\
\hline Dimensiones de los focos en hectáreas & 313.200 \\
\hline Valores actual de los terrenos palúdicos, pts. & 24.518 .750 \\
\hline Coste aproximado de las obras para sanearlos & 50.682 .500 \\
\hline Valor aproximado que tendrían saneados & 124.864 .000 \\
\hline Morbilidad anual & 301.360 \\
\hline Mortalidad Ídem & 2.192 \\
\hline Consumo anual de quinina, grs. & 2.783 .250 \\
\hline Valor de la quinina consumida al año, pts. & 2.783 .250 \\
\hline $\begin{array}{l}\text { Días de trabajo perdidos por la enfermedad, a razón de } \\
\text { quince días }\end{array}$ & 4.520 .400 \\
\hline
\end{tabular}

La intervención antipalúdica en España en el momento de la publicación de los textos estaba en sus primeras etapas. Aunque se reconocían las iniciativas de Río Tinto o la de la Mancomunidad de Cataluña, se consideraban insuficientes dada la magnitud del problema: «[...] aquí, aunque digno de elogio

47 Rodríguez; Ballester; Perdiguero-Gil; Medina; Molero (2003), p. 61-62. Ver también: Bernabeu-Mestre, J.(1997), «Cultura, ciencia y política. La lucha antipalúdica de la Cataluña de la Mancomunidad, 1914-1925», Medicina e Historia, $\mathrm{n}^{\circ} 73$.

48 MuÑOZ (1921), p.152.

49 MUÑOZ (1921), p.164. 
lo poco que se ha hecho, no se le ha dado por los gobiernos ni las autoridades locales el impulso que debiera» ${ }^{50}$. Tal y como menciona Muñoz Antuñano, en 1921 la campaña de lucha contra el paludismo ya había comenzado; al menos en Cataluña, donde de la mano del Institut d'Estudis Catalans, la Mancomunidad puso en marcha, a partir de 1915, el Servicio Técnico de Paludismo ${ }^{51}$. Pero, ciertamente, su extensión por todo el país se produjo de una forma irregular. Hasta 1920, no se creó el primer organismo central responsable de la intervención, la Comisión para el Saneamiento de las Comarcas Palúdicas, una iniciativa que no se menciona en los textos analizados. Las únicas acciones legislativas que se mencionan son: una ley encaminada al estudio del paludismo del 30 de Enero de 1900, suscrita por el entonces Ministro de la Gobernación D. Eduardo Dato; la creación de la ya mencionada Inspección para el Saneamiento del Campo, dependiente del Ministerio de Fomento; y el proyecto de Ley de 1918 para la desecación y saneamiento de marismas y pantanos, pero que siendo todavía un proyecto, ya era valorada como insuficiente ${ }^{52}$.

Aparte de estas reflexiones, en las obras analizadas también se aporta un repaso actualizado de los mecanismos fisiopatológicos de la enfermedad, así como de la forma de transmisión. Se describe la situación de la enfermedad en otros países - Italia ${ }^{53}$, Grecia, EEUU, Egipto (Canal de Suez)—, así como las estrategias utilizadas para prevenirla. Básicamente, estas estrategias se enmarcaban en dos grandes líneas de actuación. Por un lado, la destrucción del mosquito y sus larvas y, por otro, la protección de las personas frente a las picaduras. Para la destrucción del mosquito y sus larvas, se plantearon medidas como el uso de cepos y cajas, la combustión de sustancias químicas, o la intervención biológica a través de especies piscícolas como los barbos y ten$\operatorname{cas}^{54}$. La protección de las personas se basaba en medidas como el uso de caretas, mosquiteros, guantes, telas metálicas en puertas y ventanas, o plantaciones de eucaliptos, que al tiempo que protegían al crear una barrera natural, contribuían a desecar el terreno.

El paludismo, como enfermedad infecciosa vinculada al medio ambiente, suponía un importante riesgo para la salud de todas las personas que desarro-

50 MUÑOZ (1921), p.157.

51 BERNABEU-MESTRE (1997).

52 MuÑOZ(1921), p.165.

53 La descripción de la acción antipalúdica italiana es extensa y detallada. Italia había llevado a cabo una intervención a gran escala, que incluyó la protección de las viviendas, desecación de terrenos e introducción de agricultura intensiva y tratamiento gratuito con quinina, Rodríguez Ocaña; Ballester; Perdiguero-Gil, Medina; Molero (2003), p. 121.

54 MuÑOZ (1921), p.157-158. 
llaban su vida en el mundo rural. Sin embargo, la idea de que existían grupos de mayor vulnerabilidad a la enfermedad se encuentra presente en las reflexiones de ambos autores. A modo de ejemplo, y en relación al caso de Río Tinto, mencionaremos como G. de Membrillera y Muñoz Antuñano consideraron diferente el riesgo de contraer la enfermedad entre hombres, niños y mujeres, por ser estas últimas un grupo cuyas actividades estaban más vinculadas al uso del agua:

«En la comarca de Río Tinto hay un pueblo en el que solamente las mujeres y los niños pequeños se afectaban de paludismo; y tras de muchas investigaciones, el Doctor MacDonald pudo averiguar que el vivero de anofeles estaba en un pozo, a la distancia de un kilómetro de la población, al que iban a surtirse de agua potable; $\mathrm{y}$, claro es, las encargadas de estos menesteres, que a veces llevaban consigo a sus niños, eran las mujeres; y esta era la razón de que únicamente ellas [...] eran las afectadası ${ }^{55}$.

\subsubsection{Anquilostomiasis}

La anquilostomiasis, también conocida como anemia de los mineros, anemia de los países cálidos o enfermedad de los túneles, fue, junto al paludismo, una de las enfermedades infecciosas más frecuentes en el mundo rural de finales del XIX y principios del $X^{56}$. En términos generales, esta patología se caracteriza por la presencia de un parásito animal, el anquilostoma duodenalis, que al alojarse en el duodeno, genera anemia severa, fiebre y alteraciones digestivas. Parte del ciclo reproductor del parásito tiene lugar en el exterior del organismo, por lo que las condiciones del entorno del individuo son muy relevantes.

La anquilostomiasis tuvo una gran prevalencia en los países calidos y de clima tropical. En Egipto, la India colonial, Guinea, Sudamérica o Ceilán fue considerada, en las primeras décadas del siglo XX, un problema endémico, relacionado con la actividad agrícola y con las condiciones climatológicas ${ }^{57}$.

55 MuÑOZ (1921), p.158.

56 Sobre la anquilostomiasis en España en este periodo ver, RoDRÍGUEZ, E; MENÉNDEZ, A. (2006). «Higiene contra la anemia de los mineros. La lucha contra la anquilostomiasis en España (1897-1936)» Asclepio (en prensa).

57 La lucha contra la anquilostomiasis fue un problema prioritario para la Fundación Rockefeller entre 1913 y 1930 . Hasta 1920 las campañas contra la enfermedad se centraron en los territorios coloniales de Gran Bretaña, fruto de la colaboración entre la Fundación Rockefeller y la Oficina Colonial Británica. A partir de entonces, y hasta 1930, la campaña se amplió a territorios de Sudamérica y Centroamérica, con resultados irregulares. FARLEY, J. 
En España, la enfermedad también estuvo muy relacionada con el trabajo en las minas, lo que no significa que fuera considerada un problema ajeno al mundo rural ${ }^{58}$. En 1915, el número de trabajadores en el sector minero, según el Boletín de la Inspección General de Minería, era de 106.206 obreros entre hombres, mujeres y niños ${ }^{59}$. Las condiciones a que se veían sometidos estos trabajadores, caracterizadas por largas jornadas laborales, escasas medidas de seguridad y frecuentes accidentes, hicieron que sus tasas de mortalidad fueran muy elevadas ${ }^{60}$. Además, las condiciones ambientales de las minas, con elevados niveles de temperatura, de humedad, falta de ventilación y escasas medidas higiénicas, fueron el caldo de cultivo para el desarrollo del ciclo vital del parásito y la infección de los trabajadores, que a través de pequeñas erosiones, adquirían la enfermedad. El hecho de que el parásito precisara de estas condiciones ambientales para su desarrollo, justifica que se incluyera entre los problemas de salud evitables. Si se llevaban a cabo las oportunas medidas de saneamiento y protección de los trabajadores, se podría evitar el contagio y la epidemia posterior.

Las primeras medidas organizadas de lucha contra la enfermedad vinieron de la mano de la ya mencionada Inspección de Sanidad del Campo que, en 1910, abordó el estudio y la campaña destinada a evitar y extinguir la ende-

(2004) To cast out disease. A History of the Health Division of the Rockefeller Foundation (1913-1951). Oxford, University Press, p. 61-87. En España, la intervención de la Fundación Rockefeller en la lucha contra la enfermedad se centró en el escaso periodo de tiempo comprendido entre diciembre de 1924 y junio de 1926. Además, únicamente consistió en la elaboración por parte de Charles A. Bayley, médico de la Fundación, de un estudio epidemiológico sobre el terreno. En realidad este trabajo estaba encubriendo, como objetivo confidencial, el estudio de la situación general de la sanidad española para así evaluar el alcance de las futuras intervenciones de la Fundación en el país. Rodriguez-OcañA, E. (2005) Salud Pública en España. Ciencia, profesión y politica, siglos XVIII-XX. Granada, Universidad de Granada, p. 120.

58 «Fuera de la mina, el minero es un obrero rural en gran número de casos, y, en este sentido, todo cuanto decimos acerca del campo y de las viviendas rurales, en especial de las colonias agrícolas, les corresponde a éstos del modo más exacto». [MUÑOZ (1921), p.84]. El problema de la anquilostomiasis relacionada con la actividad agrícola en España se describe en DARriBA, A.R. y CÁnOVAS, M.A., Sobre anquilostomiasis en la huerta de Murcia, Madrid, Publicaciones oficiales de la CPIS. También en el texto de HERNÁNDEZ- PACHECO, D. (1927), La anquilostomiasis en la huerta de Murcia. Boletín Técnico de la Dirección General de Sanidad. Año 2, p. 573-584.

59 MuÑOZ (1921), p.71.

60 En el XIV Congreso de Higiene y Demografía celebrado en Madrid en 1898, Ricardo Gómez de Figueroa, médico de las minas de Almadén, aportaba una cifra de mortalidad del 16 por 100 [sic]. [MUÑOZ (1921), p.73]. 
mia $^{61}$. Según la Real Orden de 14 de Julio de 1911, la primera estrategia fue la elaboración de una encuesta, que se envío a las compañías mineras y en la que se obtuvieron 100 cuestionarios debidamente cumplimentados. Según esta encuesta y salvando las deficiencias y sesgos metodológicos, la enfermedad no era endémica en las minas españolas, salvo en la región de Andalucía, sobre todo en Linares, La Carolina y en algunas zonas de Almería ${ }^{62}$. Esta información, que fue confirmada tras la visita de D. Bonifacio de la Cuadra, Inspector de Sanidad del Campo en la región, permitió que se dictara una nueva disposición el 3 de Enero de 1912.

A partir de esta nueva disposición, se generó un importante conflicto entre los trabajadores y las compañías mineras, que desplazó el foco de atención de lo sanitario a lo laboral. El problema de los trabajadores surgió porque, entre las recomendaciones higiénicas, se incluyó la prohibición de bajar a las galerías a los obreros infectados por la enfermedad ${ }^{63}$. Esta medida, dada la magnitud de la infección, suponía una situación de paro forzoso entre los obreros. Los conflictos de orden público generados por esta situación, desplazaron las reivindicaciones hacia la necesidad de conseguir que la anquilostomiasis se considerara accidente del trabajo imputable a las Compañías Mineras, y se relegó a un segundo plano la extinción de la endemia. Además, los responsa-

61 Hubo algunas iniciativas científicas previas de carácter individual por parte de médicos interesados en la cuestión. En el año 1882, el Dr. Rodríguez Méndez publicó un trabajo sobre el tema en La Clínica de Zaragoza, para llamar la atención sobre el asunto a las Compañías Mineras y a los Poderes Públicos. D. Bonifacio de la Cuadra, médico de Úbeda y posteriormente inspector regional de Sanidad del Campo de Andalucía oriental, escribió una comunicación a la Real Academia de la Medicina de Madrid, comunicando un caso de anquilostomiasis en un ingeniero de minas, con el fin de animar a la institución a emprender una campaña de lucha contra de la enfermedad. También el Dr. Codina, los Srs. González y el Dr. Larra Cerezo, publicaron diversos artículos y folletos sobre el asunto [MUÑOZ (1921), p.91-92].

62 MuÑOZ (1921), p. 93-94.

63 La Disposición se resumía en seis apartados: « $1^{\circ}$ Que se remitan por las Compañías y Sociedades mineras los cuestionarios informados. $2^{\circ}$ Que por los Inspectores de Sanidad del Campo se practique un reconocimiento técnico de las minas y se propongan las medidas más oportunas para su saneamiento. $3^{\circ}$. Que las Compañias no admitan un nuevo obrero sin el previo reconocimiento de sus heces. $4^{\circ}$ Que se prohiba bajar a los obreros infectados por anquilostomiasis a la mina, para lo cual deben antes sufrir un reconocimiento del médico que deben tener las minas, según lo que dispone el artículo 23 de Policía minera. $5^{\circ}$ Que las Compañias saneen y desinfecten las galerías y pozos infectados. $6^{\circ}$ Prohibición de defecar en la mina y de que permanezcan en ella descalzos los mineros, y que se establezcan en las minas lavabos, retretes, guardarropas, y que existan retretes portátiles en su interior» [MUÑOZ (1921), p.95-96]. 
bles de las Compañías no aplicaron la legislación convenientemente, lo que fue agravando el conflicto. Los obreros, tras amenazas de paros y huelgas, iniciaron acciones individuales por vía judicial, lo que forzó una nueva Real Orden (R.O. de 9 de Agosto de 1916), que reiteraba la de 1912, e incorporaba la necesidad de habilitar laboratorios, para auxiliar la labor de los médicos de las minas y de los Inspectores de Sanidad ${ }^{64}$. En 1921, las reales Ordenes todavía no habían tenido el cumplimiento debido, pues seguían existiendo en los hospitales de Madrid, casos de mineros con anquilostomiasis procedentes de la zona minera de la Carolina ${ }^{65}$.

\subsection{La salud de la infancia en el mundo rural}

En 1904, se aprobaba en España, promovida por Manuel Tolosa Latour, la Ley de Protección a la Infancia. Esta norma, a pesar de que tuvo un alcance limitado y diversos autores así lo han puesto de manifiesto ${ }^{66}$, supuso una mejora frente al marco regulador anterior y logró la movilización de la sociedad española frente al problema de la infancia. Según su modelo de organización, junto a la creación del Consejo Superior de Protección a la Infancia en el Ministerio de Gobernación, se crearon también las Juntas Provinciales y las Juntas locales, lo que podía haber supuesto una mejora en el mundo rural. Sin embargo, tanto la falta de una financiación oficial estable ${ }^{67}$, como el hecho de que las prioridades del momento se situaran en el mundo urbano, impidieron que su alcance fuera mayor. De hecho, en los primeros informes sobre infancia de las Juntas Provinciales, el ámbito rural, no sólo no se incluye entre las prioridades de actuación, sino que, dentro de la idealización de la vida en el

64 MuÑOZ (1921), p.95-99.

65 MuÑOZ(1921), p.100.

66 Rodríguez OCAÑA, E. (1986), «Medicina y acción social en la España del primer tercio del Siglo XX». En LóPEZ, C (ed). De la beneficencia al bienestar social. Cuatro siglos de acción social. Madrid, Siglo XXI, 227-266, p.234-35. Perdiguero, E; RoBleS, E. (2004), «La protección a la infancia y la Sociedad Española de Higiene». En PERDiguero, E (ed). Salvad al niño. Estudios sobre la protección a la infancia en la Europa Mediterránea. Valencia, Seminari d'Estudis sobre la Ciencia, 93-120, p. 120. y BARONA, JL. (2004) «El Consejo Superior de Protección a la Infancia y Represión de la Mendicidad (1904-1914). Su ideología social y sanitaria». En Perdiguero, E (ed). Salvad al niño. Estudios sobre la protección a la infancia en la Europa Mediterránea, Valencia, Seminari d'Estudis sobre la Ciencia, 121-153, p. 123.

67 BARONA (2004), p.121-123. 
campo y del mundo campesino ${ }^{68}$, es visto como una solución para los niños abandonados, viciosos o delincuentes ${ }^{69}$.

Sin embargo, la vida de los niños en el campo, lejos de ser idílica, estaba sometida a las mismas condiciones de vida y de trabajo - en el mundo rural el trabajo infantil se consideraba una ayuda familiar indispensable- que sufrían los adultos, con el agravante añadido de la mayor vulnerabilidad de los menores $^{70}$. Las enfermedades infecciosas de la infancia, la explotación laboral de niños y niñas, el analfabetismo y la situación de las escuelas, o «la ignorancia sanitaria y los prejuicios antihigiénicos» ${ }^{71}$ de las familias, eran algunos de los principales problemas que requerían una solución sin demora.

Superar las enfermedades contagiosas de la niñez suponía un margen de seguridad para sobrevivir a las duras condiciones de vida del campo. No era fácil ganar la batalla a enfermedades como el sarampión, la difteria, la meningitis o la gripe, pues la vida de estos niños, se hallaba repleta de importantes condicionantes que les hacia especialmente susceptibles a las infecciones. La miseria generalizada, las deficientes condiciones higiénicas de escuelas y talleres o el exceso de trabajo, colocaba a los niños en una situación de gran vulnerabilidad ${ }^{72}$.

No menos relevante eran las condiciones de trabajo que apuntábamos en párrafos anteriores, o si se prefiere, la explotación a que estaba sometida la

68 En relación con esta idea, BERnABEu-Mestre (2005), p. 291, habla del «Mito de la salud rural». El autor, con esta expresión, hace referencia a la suposición errónea de que en las poblaciones rurales existían mejores niveles de salud y mejores condiciones de salubridad, cuando las cifras de mortalidad, al menos en el caso catalán, eran en las zonas rurales, superiores a las de la población urbana.

69 Así lo expresa BAronA (2003), p. 130, cuando analiza el Primer Informe de la Junta Provincial de Barcelona. Según el autor «los niños recogidos de la calle, se dividían en dos grupos, niños abandonados, viciosos o delincuentes, y simplemente abandonados. Los primeros debían someterse a un tratamiento educativo correccional hasta normalizarlos y trasladarlos a alguna familia rural agrícola. Los simplemente abandonados iban directamente a familias del campo, garantizadas por el párroco y el alcalde de la localidad.

70 Sobre esta cuestión ver BORRÁs, JM. (2002), «El trabajo infantil en el mundo rural español (1849-1936)». En MARTINEZ, JM (2002). El nivel de vida en la España rural, siglos XVIII-XX, Alicante, Universidad de Alicante, 497-547.

71 Algunas costumbres del mundo rural que G. DE MEMBRILLERA (1921), p. 32, calificaba como «maltrato», consistían en «dar a la criatura sendos tragos de vino, [...], y hay regiones donde para hacerlas el estomago (deshacerles, deberían decir) les dan a las criaturas a los pocos días de nacer, una copa de aguardiente, y le hacen chupar una tira de jamón [...] o la resistencia de las madres a que los pequeñuelos que amamantan les caiga el caparazón de suciedad que el abandono les forma en la cabeza».

72 G. DE MEMBRILLERA (1921), p.32-33. 
población infantil. La legislación (Ley de 13 de Marzo de 1900) ${ }^{73}$ regulaba las condiciones de trabajo de mujeres y niños; sin embargo, los contratos de servidumbre y la pobreza de las familias hacían muy difícil su cumplimiento. Así, numerosos niños y niñas, en plena etapa de desarrollo, trabajaban de pastores, sirviendo en casas ajenas, o en la recolección agrícola, lo que los sometía a esfuerzos superiores a su capacidad ${ }^{74}$.

Dedicar a los niños a estas labores implicaba sacarlos prematuramente de la escuela, contribuyendo a las altas cifras de analfabetismo ${ }^{75}$. No obstante, asistir a la escuela tampoco era una garantía de salubridad. Las condiciones de la escuela no eran mejores que las de las viviendas: la mayoría de ellas estaban ubicadas junto a las cuadras o en los graneros, muchas no tenían ventanas, carecían de agua corriente y retretes, lo que contribuía a empeorar las condiciones de vida de la infancia en el mundo rural: «[...] 27 escuelas de la provincia de Málaga tienen el vertedero de las infantiles inmundicias en el patio o corral, junto a la clase; de 429 escuelas de otra provincia, 400 carecen de agua. La inmensa mayoría de las de España están sin retrete, y más vale así, si hubieran de tenerlo, como algunas de las que no nombro, que lo abrigan dentro de la misma aula $[\ldots]{ }^{76}$

3. A MODO DE CONCLUSIÓN: ALGUNAS PROPUESTAS PARA RESOLVER EL PROBLEMA SANITARIO

Los trabajos que hemos analizado son, a nuestro juicio, dos interesantes ejemplos de cómo se integraron a finales del S. XIX y primeros del XX, en torno a la Higiene, disciplinas tan diversas como la Ingeniería y la Medicina. Esta vertebración fue posible, no sólo por el interés de los profesionales por la deficiente situación de salud de la población, que requería una acción multidisciplinar, sino por el propio concepto de la Higiene, disciplina integradora y con amplia vocación de «ciencia de gobierno» ${ }^{77}$ y cuya misión era resolver los problemas más complejos

\footnotetext{
73 BARONA (2003), p. 133.

74 MuÑOZ (1921), p. 20-21

75 Sobre la escuela rural, ver SARASÚA, C (2002), «El acceso de niñas y niños a los recursos educativos de la España rural del siglo XIX», En MARTINEZ, JM (2002). El nivel de vida en la España rural, siglos XVIII-XX, Alicante, Universidad de Alicante, 549-609.

76 MuÑOZ (1921), p. 36-37.

77 Así la calificó Menéndez Álvaro, en su discurso de ingreso en la Real Academia de Madrid en 1853. CAMPOS (1998), p.74.
} 
de las sociedades. Frente a esta misión, y amparados por el carácter científico de sus propuestas, los higienistas se sintieron claramente legitimados, en un contexto en el que la irrupción del positivismo enmarcaba su labor.

La Higiene se planteó como la solución a los problemas. Los higienistas a través de sus propuestas higiénico-morales se erigieron en expertos llamados a intervenir para lograr mejoras en las condiciones de vida de la población. Las intervenciones se articularon en dos grandes líneas de acción, por un lado, la educación - moralización de las personas, junto al saneamiento del medio, y por otro, la puesta en marcha de reformas legislativas y administrativas en el espacio público español. También fue relevante la consideración de la deficiente situación socioeconómica de la población, que en el caso del mundo rural, presentaba condicionantes distintos a los del mundo urbano, y requería, por tanto, adoptar una perspectiva diferente e intentar la puesta en marcha de planes específicos.

Las dos vertientes fundamentales de la acción higiénica, la educación ${ }^{78}$ - moralización de las clases populares - y el saneamiento del medio, estuvieron íntimamente ligados, aunque se ha podido identificar en los textos el diferente peso de cada una de ellas según el contexto. En el ámbito rural el saneamiento del medio se erige como el objetivo principal. La educación, pero sobre todo la moralización, era una cuestión más vinculada al mundo urbano. La degeneración social, manifestada en cuestiones como la prostitución, la miseria, las enfermedades de transmisión sexual, el alcoholismo, etc., era más propia de las ciudades, y era el elemento que, a juicio de los higienistas, más requerimientos de moralización estaba precisando. Los problemas del mundo rural, se vincularon más al medio. A los campesinos, aunque también se les atribuye su parte de culpa, se les ve más como victimas de su entorno, que como personas degeneradas e inmorales.

La responsabilidad de las iniciativas legislativas y de planificación, por su parte, recaía en el Estado, quien debía dictar, ordenar y, en gran medida, ejecutar los planes de saneamiento nacional. Era preciso reorganizar los servicios sanitarios y el régimen administrativo español, ya que no permitía interrelacionar departamentos, direcciones o negociados, y mostraba graves incoherencias, al gastar importantes sumas de dinero en obras de ingeniería, que no estaban asociadas a objetivos sanitarios ${ }^{79}$.

78 Los Municipios podían colaborar en la consecución de estos objetivos educativos a través de acciones sanitarias como la concesión de premios en las escuelas a los alumnos que se distinguieran por su limpieza, o a los propietarios de los hogares más limpios e higiénicos [G. DE MEMBRILleRA (1921), p. 94].

79 Para llevar a cabo tal reorganización se proponían como medidas prioritarias la creación de un Ministerio o Dirección de Sanidad y la puesta en marcha de una estadística sanita- 
Los condicionantes socioeconómicos de la población rural señalaban la necesidad de acciones globales que incidieran en las condiciones de vida de las personas. La reorganización de la propiedad de la tierra, la colonización interior como instrumento para lograr propiedades rústicas y huir de la esclavitud del arrendamiento, la mejora de los salarios o la reducción del gran abismo entre los muy ricos y los muy pobres, fueron medidas que se plantearon como necesarias ${ }^{80}$.

La vida en el medio rural, no por estar vinculada a la naturaleza, era una vida sana y repleta de beneficios. El entorno rural también podía ser insalubre y estaba vinculado a problemas sanitarios muy graves. La idea de que el mito de la salud rural estaba lejos de ser realidad se hace también patente en los textos de Membrillera y Muñoz Antuñano.

Fecha de recepción: 5 de abril de 2006

Fecha de aceptación: 16 de junio de 2006

ria de todos lo pueblos de España. También se consideró fundamental la dotación de recursos a los organismos implicados, tanto económicos, como profesionales para poder poner en marcha los planes propuestos [G. DE MEMBrillera (1921), p. 91-92]. Sobre la creación del Ministerio de Sanidad, ver HUERTAS, R (1993), «El debate sobre la creación del Ministerio de Sanidad en la España del primer tercio del siglo XX. Discurso ideológico e iniciativas políticas», Asclepio, 45 (1) 89-122.

80 A todo esto, podía ayudar, en cierta medida, el fomento de instituciones agrarias de carácter económico y financiero, que bajo el nombre de Sindicatos Agrícolas o Cajas Rurales contribuyeran con créditos y ayudas a mejorar la situación económica de los labradores. Así, se frenaría la emigración rural y se favorecería la adquisición y el cultivo de las pequeñas propiedades [MUÑOZ (1921), p. 144]. 
